\title{
Progress in Luminescent Solar Concentrator Research: Solar Energy for the Built Environment
}

\author{
Paul P. C. Verbunt ${ }^{1}$, Michael G. Debije ${ }^{1 *}$, \\ ${ }^{1}$ Eindhoven University of Technology, Eindhoven, The Netherlands \\ * Corresponding author: Tel: +31 40 2475881, Fax: +31 40 2436999, E-mail: mgdebije@tue.nl
}

\begin{abstract}
This paper presents a concise review of recent research on the luminescent solar concentrator (LSC). The topics covered will include studies of novel luminophores and attempts to limit the losses in the devices, both surface and internal. These efforts include application of organic and inorganic-based selective mirrors which allow sunlight in but reflect emitted light, luminophores alignment to manipulate the emitted light path, and patterning of the dye layer. Finally, the paper will offer some possible 'glimpses to the future', and offer some additional research paths that could result in a device that could make solar energy a ubiquitous part of the built environment as sound barriers, bus stop roofs, awnings or siding tiles. Considering the reported efficiencies of the LSC are comparable to those reported for organic PVs, which are also being considered for use in the built environment, the results of the research on the LSC to date warrants more widespread attention.
\end{abstract}

Keywords: Solar energy, Luminescent solar concentrator, Building integrated photovoltaics, Review

\section{Introduction}

The European Committee wants all newly built buildings to be near-zero energy by $2020^{[1]}$. This demands architects integrate energy saving and energy generation into their designs. To give architects more freedom, the devices saving and/or generating energy must be easily adaptable. A readily available energy source in a built environment is the sun, which is clean, safe, inexhaustible and reliable but generation of electricity with conventional photovoltaic (PV) cells has several disadvantages in this environment: the cells remain costly, modules are heavy, and limited in coloration (black and dark blue). Furthermore, PV cells respond optimally to direct sunlight, while in the built environment much of the sunlight is diffuse due to scattering and reflections by other objects, such as trees, buildings, and even clouds.

An alternative solar energy harvester was proposed in the 1970's, the luminescent solar concentrator $(\mathrm{LSC})^{[2]}$. In the LSC, sunlight penetrates the top surface of an inexpensive plastic or glass waveguide. This light is absorbed by luminescent molecules which are either embedded in the waveguide or applied in a separate layer on top or bottom of the waveguide. The luminescent molecules can be organic fluorescent dyes, or inorganic phosphors or quantum dots. The absorbed light is re-emitted at longer wavelengths, and a fraction of the re-emitted light is trapped in the waveguide by total internal reflection and becomes concentrated along the edges of the plate. Small PV cells attached to edges of the waveguide collect the emission light and convert it to electricity.

The LSC has potential advantages over silicon-based PV panels, especially in the built environment. For one, they can reduce the size of the PV cells more than $90 \%$ and the materials for making LSCs are inexpensive, reducing module prices. The plastic waveguide is lighter than the silicon PV panels, leading to a reduction in weight, which makes LSCs more viable for mounting to the sides of buildings. Sunlight can better penetrate the surface of the LSC waveguide from all angles, making them more appropriate for collecting non-direct sunlight. Lastly, LSCs have a great flexibility in design, which make them attractive for architects: they may be made thin and can be cut in any desired shape, cast in almost any color and may be transparent. Since LSCs can be thin, it becomes possible to make curved device. If the photon in/ photon out efficiency is high enough, the cost of electricity generated by the LSC could be competitive ${ }^{[3]}$. These combined features make 
LSCs interesting devices for increasing public acceptance of solar energy: while the efficiency of an LSC will be lower than an equivalent area of a silicon PV, the reduced cost and flexibility in design could make LSCs viable for the urban area. The LSC hasn't yet been commercialized owing to several drawbacks which limit their efficiency ${ }^{[4]}$. Loss mechanisms for LSCs are shown in Figure 1.

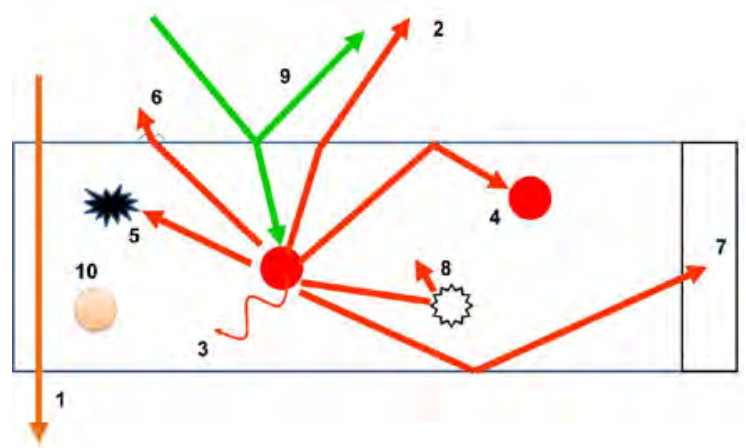

Figure 1. Loss mechanisms in LSCs: 1) Input light not absorbed by the dye molecules, 2) Light emitted outside capture cone, 3) Quantum efficiency of the dye molecules <1, 4) Re-absorption of emitted light by another dye molecule, 5) Absorption of emitted light by the waveguide, 6) Surface scattering, 7) Solar cell losses, 8) Internal waveguide scattering, 9) Reflection from the e surface, 10) Limited dye stability.

The first loss is sunlight not absorbed by the dye molecules: this light is lost through the bottom surface. The second is light emitted by dye molecules under an angle which is refracted out of the waveguide instead of reflected internally- ( $>40 \%$ of all absorbed energy may be lost through the top and bottom surfaces of the LSC ${ }^{[5]}$ ). The third loss is absorbed photons not re-emitted by the dye molecules, but instead lost as heat and vibrations. Re-absorption of emitted photons by subsequent dye molecules via overlap of emission and absorption bands is a fourth loss. Waveguides can exhibit parasitic absorption, especially in the near infrared, and is the fifth loss cited. Sixth, imperfections of the waveguide surface can cause photons in the waveguide mode to leave the surface. Seventh, the PV cell at the waveguide edge has a non-uniform spectral response, with a fraction of incident photons being lost due to the finite conversion efficiency. Imperfections in the waveguide bulk lead to the eighth loss, scattered waveguided photons. In addition, a small part of the input light is reflected from the surface of the waveguide, shown as the ninth loss. Finally, there is loss caused by degradation of the dye molecules, primarily due to UV absorption.

\section{Losses and Proposed Solutions}

\subsection{Surface Loss}

Dye-emitted photons emitted inside the escape cone will be lost through the surfaces, and measurements suggest that $40-55 \%$ of all absorbed energy is lost in this way (this translates into a $50-70 \%$ loss of photons) ${ }^{[5]}$ : these results were confirmed by simulation ${ }^{[6]}$. This surface loss is a key one for LSCs, and in the last couple of years multiple groups have done research on minimizing them by two processes: aligning the luminophores and applying selective mirrors.

Aligned luminophores: Organic luminophores are often dichroic in absorption and transmission ${ }^{[7]}$, opening new possibilities in controlling the spatial distribution of emitted light, provided that the physical ordering of the dyes is macroscopically controlled. The alignment of dichroic dyes in liquid crystalline (nematic) materials has been previously investigated ${ }^{[8]}$ and it was shown that the macroscopic alignment of the dyes in the liquid crystalline host resulted in anisotropy and dichroism in both absorption and emission. Aligning the dye luminophores perpendicular to the waveguide surface leads to an emission primarily in the direction of the waveguide, resulting in a sharp decrease of surface loss to less than $10 \%{ }^{[9]}$,confirmed by simulations using collimated light ${ }^{[6]}$. 
However, in this configuration the luminophores have low absorption, and concurrent low edge emission: trapping efficiency of emitted photons increases from $~ 65 \%$ to over $80 \%$ when vertically aligned luminophores in LSCs are excited by an isotropic light source ${ }^{[10]}$.

Luminophores can also be aligned planarly, or parallel to the waveguide surface. This configuration can direct light so that $60 \%$ more energy is emitted from two edges of the LSC compared to the other two edges ${ }^{[11]}$ : in this way the LSC can be used as a energy harvesting polarizer ${ }^{[12]}$ which could be used in displays, for example. Additionally, if light is emitted primarily towards just two edges, the number of PV cells on the LSC can be reduced to two or even one, further reducing LSC cost.

Selective mirrors: A second way to reduce surface loss is by applying wavelength-selective mirrors [7, 21-31]. These mirrors are placed on top the LSC-waveguide with the goal that the reflectors not interfere with incoming sunlight that can be absorbed by the luminophores, but reflect only the luminophore-emitted light, which has a longer wavelength. Wavelength selective mirrors, made from chiral nematic (cholesteric) liquid crystals ${ }^{[13]}$ or inorganics ${ }^{[13 b, 15]}$, have both been applied to the LSC. Up to $30 \%$ of the light that had previously escaped the surface was turned into edge emission, translating into a $12 \%$ LSC output improvement using the organic reflectors ${ }^{[14 f]}$. Similar enhancements were determined using the inorganic reflectors ${ }^{[4 b]}$. Organic reflectors are cast from solution and spontaneously form the reflective layer: this is generally a much simpler and less expensive process than application of multilayer inorganic Bragg reflectors.

\subsection{Re-absorption of emitted photons by other dye molecules}

Most organic luminophores used in LSCs have small Stokes-shifts, leading to relatively large overlaps between the absorption and the emission spectrum ${ }^{[15]}$. As a consequence, luminophoresemitted photons can be re-absorbed by another luminophore molecule during transportation through the waveguide. Re-absorptions are not losses by themselves, but the limited quantum efficiency of the luminophores and re-emission into the escape cone do result in losses ${ }^{[16]}$.

To reduce re-absorption events, researchers have experimented with luminophores with large Stokes-shift, like lanthanides ${ }^{[17]}$ and quantum dots ${ }^{[18]}$,but these classes of luminophore bring other challenges to the production of LSCs. Inorganics tend to suffer from low solubility in organic matrices, and often also suffer from a low absorption. To reduce the amount of reabsorptions Taleb et al. ${ }^{[19]}$ doped a dye with a polar and highly mobile material, like thionin. The dopant increased the separation of the absorption and fluorescence bands of the dye molecules, increasing the Stokesshift and reducing (but not eliminating) the overlap in absorption and emission spectrum.

An option to reduce encounters of emitted light with the dyes is to only attach the dyes in a thin layer at the surface of the waveguide rather than filling the luminophore within the bulk of the waveguide. In this way, emission light may be transported predominantly in the clear host material, and only encounter the dye layer again every second internal reflection. These layers have been made of acrylates, via sol-gel techniques ${ }^{[20]}$, and polymerized liquid crystals ${ }^{[11]}$.

Using spatially-separated patterns of luminophores on top of a waveguide, the number of reencounters emitted light could have with other dye molecules was reduced ${ }^{[21]}$. The transport efficiency of the photons through the LSC increased with decreasing dye coverage. However, due to reduction in absorption, the total system output decreased. A lens system on top of the LSC is being developed that would increase absorption and thus the system output ${ }^{[22]}$. 


\subsection{Dyes: Limited absorption, stability and fluorescence efficiency}

The spectral breadth of the dye absorption is an important factors determining the potential efficiency of the LSC waveguide. There are a number of luminescent materials being studied for possible inclusion in the LSC. The workhorse of the organic dyes are based on perylenes or perylene derivatives ${ }^{[23]}$. The limitation of the organic dyes are often their lifetimes of operation in sunlight. Numerous studies are somewhat inconclusive as to the photostability of these materials: much depends on the processing conditions and polymeric environment of the fluorophore ${ }^{[24]}$. Organo-metallic molecules with good photostability (such as porphyrins) have also been proposed $^{[25]}$. Inorganics such as lanthanides ${ }^{[17]}$ hold promise as long-lasting replacements for organics, possibly with extended Stokes shifts. However, they tend to suffer from decreased absorption, and solubility becomes a definite issue. There is a large research effort directed at using quantum dots in the $\operatorname{LSC}^{[18]}$, but they have not yet reached their promise as they continue to generally display small Stokes-shifts with limited photostability. A possible future research direction is into the use of surface plasmonics to enhance the emission of the dye materials, allowing the use of potentially lower quantum efficiency luminophores ${ }^{[26]}$.

To aid in luminophore absorption, it is standard practice to apply a rear layer to an LSC to act as a reflector. The reflecting back layer effectively doubles the path length of incident light through the dye layer for enhanced absorption. Some of the initial experiments used a silver mirror ${ }^{[2 b]}$, but such a mirror is absorbing in the visible range. To avoid absorptive losses, most recent work has employed a white scatterer ${ }^{[4,27]}$. When separated from the waveguide by a small air gap, the rear scatterer can provide additional light for waveguides least $35 \mathrm{~cm} \mathrm{long}{ }^{[28]}$. The scatterer also may direct that fraction of incident light that cannot be absorbed by the dye directly at the PV cell, allowing it to generate electricity. The separation of the scatterer from the waveguide by a low refractive index layer is important to maintain waveguided light in the trapping modes of the waveguide: every encounter with the attached scattering layer re-distributes the light, and a significant fraction of this re-directed light will be outside the waveguide modes of the system.

Another option is to allow the LSCs to be transparent. In this form, the device could be used as a window while generating electrical current. An alternative design uses, rather than a waveguide with embedded, inflexible dyes, two glass plates coated with a conductor, the space between being filled by a liquid crystal containing a dye molecule ${ }^{[29]}$. The liquid crystal can be continually switched between orientations, from planar to homeotropic through application of a voltage across the plates. In the former state, the dye molecules follow the alignment of the LC host, and into a position of maximal absorption. The dye may then emit light which is partially trapped in the glass panes making up the 'window', and generate electrical current. By switching from planar to a tilt configuration, the output of the window necessarily drops due to reduced light absorption, but still produces a current, and the efficiency of edge output is actually increased. This design, while still needing considerable work to get transmissive properties correct with acceptable coloration, has advantages over other 'smart' windows. Photo- and thermochromics ${ }^{[30]}$ or standard blinds ${ }^{[31]}$ can, for example, be automatically switched between light and dark states, but generate no electricity. Thin-film PV modules can generate electricity ${ }^{[32]}$, but cannot have their transparency switched.

\subsection{Photovoltaic losses}

The standard silicon-based PV has a band gap corresponding to a photon of around $1100 \mathrm{~nm}$ $(\sim 1.1 \mathrm{eV})$. Photons with energies above this threshold may still be processed by the solar cell, of course, but the excess energy of the photon is wasted, and converted most often into heat, and there is a reduction in the response of the cell for these shorter wavelengths. However, the LSC does not emit a spectrum remotely similar to the solar spectrum. Rather, it emits a narrow range of wavelengths, most-often centered at red and near-infrared wavelengths $(630-720 \mathrm{~nm}$ at the 
maximum). To better exploit the spectrum of the LSC, researchers have used type III-V PV cells based on GaAs and InGaP cells ${ }^{[4 a]}$ and obtained record-setting efficiencies. If these cells could be produced economically, it could hold great promise for widespread adoption of the LSC in future. Another option could be the use of organic-based PV cells, which often have a 'sweet spot' in the spectral range where the LSC emits ${ }^{[33]}$.

\subsection{Waveguide losses}

Around $4 \%$ of incoming light is reflected from the waveguide surface (the refractive indexes of polymethylmethacrylate (PMMA) and polycarbonate (PC) being between about 1.49 and 1.59) and never enter the waveguide, and could thus be considered a loss. While anti-reflection coatings are very common in PV cells, they have not yet been applied to LSCs. As the LSC relies on total internal reflection from two smooth surfaces, textured systems as used in many antireflective coatings $^{[34]}$ are not a viable option. Rather, coatings utilizing differences refractive indices can reduce these reflective losses and can be applied to polymeric materials ${ }^{[35]}$.

One challenge to produce luminophores with emissions approaching $800 \mathrm{~nm}$ for use in LSCs is that the waveguides, which are predominantly made of PMMA or PC, become parasitic, and absorb strongly at these wavelengths ${ }^{[36]}$. Additionally, additives made for improvement of various characteristics of the host matrix (such as altering UV stability or hardness) can have large impact on the device's capability of transporting light. For example, an additive that only shows a small absorption when measured through the width of the waveguide can have a severe impact on the edge output of the same object, for the pathlength is magnified many tenfold ${ }^{[37]}$. As waveguides age, UV-generated damage creates light 'traps' within the polymer. Research into co-polymer systems has demonstrated enhanced photostability over the single component ${ }^{[38]}$. Non-uniform edge emission from waveguides also causes additional losses, as illumination of the attached PV at anything less than uniformity results in decreased performance. Thus, the shape of the waveguide also influences the emitted light distribution ${ }^{[39]}$.

\section{Future directions}

There are a great number of improvements that can and need to be made on the LSC to make it a more viable option for use in the urban environment. One aspect we find particularly intriguing is to provide an opportunity for the use of organic-based photovoltaics (OPV). One of the greatest challenges for OPV has been the inability of utilizing the ultraviolet portion of the UV spectrum, as well as survive the high energies of the UV light which causes premature degradation of the OPVs through destruction of the dye materials. However, the LSC does not illuminate the attached solar cell with a solar spectrum, but a much more narrow-band of light, generally in the near infrared, the range of wavelengths where OPVs perform their best. Coupled with the lack of exposure to UV light, this could provide the OPV with the first real niche application where they could excel.

A second largely unexploited research area is in the field of plasmonics. Many research publications show when a fluorescent molecule is brought close to a small metallic nanoparticle there is an enhancement of the fluorescence ${ }^{[40]}$. There has been application of surface plasmonics in $\mathrm{PVs}^{[41]}$, but to our knowledge, no extensive work in the field of LSCs.

It is the opinion of the authors that the rôle of the LSC in future urban renewable energy plan should be re-defined. Given the decrease in the costs associated with traditional silicon-based PV, it would seem folly to attempt to compete directly with the well-established, traditional PV panel on a rooftop. Rather, the LSC could best be used as a complement to silicon PV rather than a competitor, positioning itself in areas not normally accessible, such as areas with increased fractions of diffuse 
light. The LSC is to be brought directly into public view, not 'hidden away' as most silicon PV panels. Applications could include sound barriers, telephone poles, and bus stop roofing.

\section{References}

[1] Directive 2010/31/EU of the European parliament and of the council of 19 May 2010 on the energy performance of buildings, Vol. 2010.

[2] a) W. H. Weber and J. Lambe, Luminescent greenhouse collector for solar radiation Applied Optics 15, 1976, 2299, b) A. Goetzberger and W. Greube, Solar energy conversion with fluorescent collectors, Applied Physics A: Materials Science \& Processing 14, 1977, 123.

[3] E. Bende et al., Proc. of the $23^{\text {rd }}$ European PV and Solar Energy Conference 2008, 461.

[4] a) L. H. Slooff, et al., A luminescent solar concentrator with 7.1\% power conversion efficiency Physica Status Solidi (RRL) - Rapid Research Letters 2, 2008, 257.

[5] M. G. Debije et al., Measured surface loss from luminescent solar concentrator waveguides, Applied Optics 47, 2008, 6763.

[6] S. McDowall, et al., Simulations of luminescent solar concentrators: Effects of polarization and fluorophore alignment, Journal of Applied Physics 108, 2010, 053508.

[7] a) M. van Gurp and Y. K. Levine, Determination of transition moment directions in molecules of low symmetry using polarized fluorescence. I. Theory, The Journal of Chemical Physics 90, 1989, 4095, b) C. Sanchez et al., Polarized photoluminescence and order parameters of in situ photopolymerized liquid crystal films, Journal of Applied Physics 87, 2000, 274.

[8] a) G. H. Heilmeier and L. A. Zanoni, Guest-host interactions in nematic liquid crystals. A new electro-optic effect Applied Physics Letters 13, 1968, 91, b) R. L. van Ewyk et al., Anisotropic fluorophores for liquid crystal displays Displays 7, 1986, 155, c) H.-W. Schinidt, Dichroic dyes, and liquid crystalline side chain polymers Advanced Materials 1, 1989, 218.

[9] P. P. C. Verbunt, D. J. Broer, C. W. M. Bastiaansen and M. G. Debije, The effect of dyes aligned by liquid crystals on luminescent solar concentrator performance, Proc. of the $24^{\text {th }}$ European PV Solar Energy Conference 2009, 381.

[10] C. L. Mulder et al., Dye alignment in luminescent solar concentrators: I. Vertical alignment for improved waveguide coupling, Optics Express 18, 2010, A79.

[11] P. P. C. Verbunt et al., Controlling Light Emission in luminescent solar concentrators through use of dye molecules aligned in a planar manner by liquid crystals, Advanced Functional Materials 19, 2009, 2714.

[12] C. L. Mulder et al., Dye alignment in luminescent solar concentrators: II. Horizontal alignment for energy harvesting in linear polarizers, Optics Express 18, 2010, A91.

[13] a) M. G. Debije, et al., Using selectively-reflecting organic mirrors to improve light output from a luminescent solar concentrator, Proc. of the WREC IX 2006, b) J. C. Goldschmidt et al., Theoretical and experimental analysis of photonic structures for fluorescent concentrators with increased efficiencies, Physica Status Solidi (a) 205, 2008, 2811, c) M. G. Debije et al., Effect on the output of a luminescent solar concentrator on application of organic wavelength-selective mirrors, Applied Optics 49, 2010, 745.

[14] B. S. Richards, A. Shalav and R. P. Corkish., A low escape-cone-loss luminescent solar concentrator, Proc. of the $19^{\text {th }}$ European PV Solar Energy Conference, 2004. 
[15] S. A. El-Daly and S. Hirayama, Re-absorption and excitation energy transfer of N,N'-bis(2,5-di-tertbutylphenyl)-3,4:9,10-perylenebis(dicarboximide) (DBPI) laser dye, Journal of Photochemistry and Photobiology A: Chemistry 110, 1997, 59.

[16] a) R. W. Olson, et al., Luminescent solar concentrators and the reabsorption problem, Applied Optics 20, 1981, 2934, b) R. Sóti et al., Photon transport in luminescent solar concentrators, Journal of Luminescence 68, 1996, 105, c) L. R. Wilson et al., Characterization and reduction of reabsorption losses in luminescent solar concentrators, Applied Optics 49, 2010, 1651.

[17] B. C. Rowan, et al., Visible and near-infrared emitting lanthanide complexes for luminescent solar concentrators, Proc. of the 24th European PV Conference 2009, 346.

[18] a) A. J. Chatten et al., A new approach to modelling quantum dot concentrators, Solar Energy Materials and Solar Cells 75, 2003, 363, b) S. J. Gallagher, et al., Quantum dot solar concentrators: Electrical conversion efficiencies and comparative concentrating factors of fabricated devices, Solar Energy 81, 2007, 813, c) V. Sholin, et al., Semiconducting polymers and quantum dots in luminescent solar concentrators for solar energy harvesting, Journal of Applied Physics 101, 2007, 123114 , d) G. V. Shcherbatyuk et al., Viability of using near infrared PbS quantum dots as active materials in luminescent solar concentrators, Applied Physics Letters 96, 2010, 191901.

[19] A. M. Taleb, Self absorption treatment for the luminescent solar concentrators, Renewable Energy 26, 2002, 137.

[20] R. Reisfeld, Fluorescent Dyes in Sol-Gel Glasses, Journal of Fluorescence 12, 2002, 317.

[21] S. Tsoi, et al., Patterned dye structures limit reabsorption in luminescent solar concentrators, Optics Express 18, 2010, A536.

[22] S. Tsoi, C. W. M. Bastiaansen and M. G. Debije, Enhancing light output of fluorescent waveguides with a microlens system, Proc. of the $24^{\text {th }}$ European PV and Solar Energy Conference 2009, 377.

[23] a) R. Reisfeld, et al., Photostable solar concentrators based on fluorescent glass films, Solar Energy Materials and Solar Cells 33, 1994, 417, b) G. Seybold and G. Wagenblast, New perylene and violanthrone dyestuffs for fluorescent collectors, Dyes and Pigments 11, 1989, 303, c) M. G. Debije et al., A promising fluorescent dye for solar energy conversion based on a perylene perinone, Applied Optics 50, 2011, 163.

[24] a) I. Baumberg, et al., Effect of polymer matrix on photo-stability of photo-luminescent dyes in multi-layer polymeric structures, Polymer Degradation and Stability 73, 2001, 403, b) R. Kinderman et al., Performance and stability study of dyes for luminescent plate concentrators, Journal of Solar Energy Engineering 129, 2007, 277.

[25] M. J. Currie et al., High-efficiency organic solar concentrators for photovoltaics, Science 321, 2008, 226.

[26] a) W. R. Holland and D. G. Hall, Waveguide mode enhancement of molecular fluorescence, Optics Letters 10, 1985, 414, b) H. R. Wilson, Fluorescent dyes interacting with small silver particles; a system extending the spectral range of fluorescent solar concentrators, Solar Energy Materials 16, 1987, 223, c) K. Aslan, et al., Metal-enhanced fluorescence from plastic substrates, Journal of Fluorescence 15, 2005, 99.

[27]a) K. Heidler, Efficiency and concentration ratio measurements of fluorescent solar concentrators using a xenon measurement system, Applied Optics 20, 1981, 773, b) J. Roncali and F. Garnier, New luminescent back reflectors for the improvement of the spectral response and efficiency of luminescent solar concentrators, Solar Cells 13, 1984, 133. 
[28] M. G. Debije et al., The effect of a scattering layer on the edge output of a luminescent solar concentrator, Solar Energy Materials and Solar Cells 93, 2009, 1345.

[29] M. G. Debije, Solar energy collectors with tunable transmission, Advanced Functional Materials 20, 2010, 1498.

[30] a) C. G. Granqvist, Oxide electrochromics: Why, how, and whither Solar Energy Materials and Solar Cells: Selected Papers from the Seventh International Meeting on Electrochromism (IME-7), 92, 2008, pp. 203, b) C. Bechinger and B. A. Gregg, Development of a new self-powered electrochromic device for light modulation without external power supply, Solar Energy Materials and Solar Cells 54, 1998, 405.

[31] M.-C. Dubois, Solar Shading and Building Energy Use, Ph.D. Thesis, Lund Institute of Technology, Lund, Sweden, 1997, pp. 1-100.

[32] a) J. Yoon et al., Ultrathin silicon solar microcells for semitransparent, mechanically flexible and microconcentrator module designs, Nature Materials 7, 2008, 907, b) P. Fath, H. Nussbaumer and R. Burkhardt, Industrial manufacturing of semitransparent crystalline silicon POWER solar cells, Solar Energy Materials and Solar Cells 74, 2002, 127.

[33] A. W. Hains, et al., Molecular semiconductors in organic photovoltaic cells, Chemical Reviews 110, 2010, 6689.

[34] S. Chattopadhyay et al., Anti-reflecting and photonic nanostructures, Materials Science and Engineering: R: Reports 69, 2010, 1.

[35] U. Schulz, Review of modern techniques to generate antireflective properties on thermoplastic polymers, Applied Optics 45, 2006, 1608.

[36] a) G. W. Chantry et al., Far infrared and millimetre-wave absorption spectra of some low-loss polymers, Chemical Physics Letters 10, 1971, 473, b) H. Ma, et al., Polymer-based optical waveguides: materials, processing, and devices, Advanced Materials 14, 2002, 1339.

[37] M. J. Kastelijn, et al., Influence of waveguide material on light emission in luminescent solar concentrators, Optical Materials 31, 2009, 1720.

[38] A. F. Mansour, Photostability and optical parameters of copolymer styrene/MMA as a matrix for the dyes used in fluorescent solar collectors, Polymer Testing 23, 2004, 247.

[39] a) M. Sidrach de Cardona et al., Edge effect on luminescent solar concentrators, Solar Cells 15, 1985, 225.

[40] T. K. Sau et al., Properties and applications of colloidal nonspherical noble metal nanoparticles, Advanced Materials 22, 2010, 1805.

[41] V. E. Ferry, et al., Design considerations for plasmonic photovoltaics Advanced Materials 22, 2010, 4794. 